\title{
Neumonía adquirida en la comunidad: recomendaciones prácticas
}

\section{Community acquired pneumonia: practical recommendations}

Moisés Morejón García

Palavras-chave: neumonía, antibióticos, infección respiratoria.
Key words: pneumonia, antibiotics, respiratory infection.

\section{Resumen}

Introducción: las infecciones respiratorias agudas y en especifico la neumonía adquirida en la comunidad, forman parte de las principales causas de consultas e ingresos en nuestros hospitales. También están consideradas como una de las principales causas de uso inadecuado de antibióticos.

Materiales y métodos: se realiza una revisión de los principales consensos internacionales sobre diagnóstico y tratamiento de la neumonía adquirida en la comunidad.

Conclusiones: todos los consensos internacionales tienen recomendaciones similares, tanto en la clasificación de los pacientes como en el tipo de terapéutica inicial.

\begin{abstract}
Introduction: acute respiratory infections (ARI) and particularly, community-acquired pneumonia are among the main causes of visiting the doctor's and admissions to hospitals. ARI are also regarded as one of the main causes of inappropriate use of antibiotics.

Materials and methods: a review of the main international consensus on diagnosis and treatment of community-acquired pneumonia is carried out.

Conclusions: all international consensus have similar recommendations, both in the classification of patients and in the type of initial therapy.
\end{abstract}

Medicina Interna. Clínico-infectólogo. Presidente APUA-Cuba. Miembro API. Hospital

Universitario

CMDTE,

Manuel Fajardo,

Ciudad Habana.

Correo electrónico: moisesm@infomed.sld.cu

Recibido:

1 de diciembre de 2011

Aceptado: 29 de diciembre de 2011

\section{Introducción}

Las infecciones respiratorias agudas y en especifico la neumonía adquirida en la comunidad, forman parte de las principales causas de consultas e ingresos en nuestros hospitales. También están consideradas como una de las principales causas de uso inadecuado de antibióticos.

\section{Desarrollo}

El tipo de tratamiento de la neumonía adquirida en la comunidad (NAC) así como el lugar de aplicación, dependerá de múltiples factores. Existen en esta patología varias clasificaciones o escalas pronósticas, las cuales utilizan disimiles parámetros, algunos que coinciden y otros no, por tal razón muchos autores consideran que todas tiene ventajas y limitaciones. A pesar de que la respuesta inflamatoria individual, crucial en la resolución y resultados, no esta incluida en las escalas pronosticas, es indiscutible que se impone la aplicación de alguna de ellas, para de esta forma tener un pronóstico y orientación en la conducta a seguir. 
De las múltiples clasificaciones que existen:

- Infectious disease society of American/ American Thoracic Society (IDSA/ATS) EE.UU(1).

- Pneumonia Severity Index (PSI) EE.UU. (2).

- CURB / CURB 65 / CURB- Edad (confusion, urea, respiratory rate, systolic or diastolic blood pressure, and age) Británico ${ }^{(3,4)}$.

- SMART-COP (systolic blood pressure, multilobar chest radiography involvement, albumin level, respiratory rate, tachycardia, confusion, oxygenation, and arterial $\mathrm{pH}$ ) Australia(5)

- CURXO-80 (España) (6)

- $\operatorname{SCAP}^{(7)}$ (Severe Community-Acquired Pneumonia)

Las más utilizadas internacionalmente son: PIS y el CURB-65.

La primera con 20 parámetros (clínicohumorales) que por su extensión y su necesidad de tecnología (gasometría-ionograma) consideramos no es práctica para su utilización en cualquier servicio de urgencia, no obstante, de poder aplicarse sería muy útil, pues basado en un resultado numérico a partir de los datos que recoge, nos da un pronósticos de mortalidad y con ello una recomendación de donde debe ser tratado el paciente (ambulatorio-sala general-Unidad cuidados intensivos).

El CRB/CURB-65 que tiene en cuenta, estado de conciencia, frecuencia respiratoria, presión arterial, urea y edad ${ }^{(8)}$, podría adolecer de que no incluye las comorbilidades del paciente, la saturación de $\mathrm{O}_{2}$ y otros, pero por otra parte es muy sencilla, práctica y también nos da una orientación pronóstica con su correspondiendo recomendación terapéutica.

A través de esta escala podemos ubicar al paciente con NAC en uno de tres grandes grupos:

Grupo I: pacientes que no necesitan ingreso (ambulatorio).

Grupo II: pacientes con criterios de ingreso pero pueden permanecer en una sala de cuidados generales.

Grupo III: pacientes que por su gravedad deben ingresar en una unidad de cuidados intensivos.
Existe cierta correlación entre cada grupo y las posibilidades etiológicas de la NAC, de ahí las distintas recomendaciones terapéuticas.

Por ejemplo los pacientes del Grupo I, son pacientes menores de 60 años sin enfermedades asociadas y sin compromiso hemodinámico, cuyas posibilidades etiológicas no sobrepasan; Streptococcus pneumoniae, Haemophilus influenzae, Chlamydia pneumoniae, Mycoplasma pneumoniae y los virus respiratorios.

El Grupo II, son pacientes que tienen más de 60 años con alguna enfermedad asociada (diabetes mellitus, enfermedad obstructiva crónica, insuficiencia cardiaca, insuficiencia renal, etc), pero que están hemodinámicamente estables y sin compromiso respiratorio importante. Este tipo de paciente solo por su edad se considera que tienen cierta senectud inmunológica, si le sumamos una o varias de las enfermedades mencionadas que también comprometen su inmunidad, tendremos un paciente expuesto a un mayor número de agentes etiológicos, como son, además de los anteriores: los bacilos gramnegativos (Klebsiella pneumoniae, Pseudomona aeruginosas, Moraxella catarrhalis, Legionella pneumophila), Staphylococcus aureus, bacteroides, $y \operatorname{otros}^{(9,10)}$.

Por último, los pacientes del Grupo III, son pacientes graves, con compromiso hemodinámico, pronósticos de mortalidad entre $30-50 \%$, que con rango similar de etiología al grupo anterior, tienen menos posibilidades de sobrevivir una terapia empírica inicial inefectiva. Hoy en día se le ha dado mucha importancia a este aspecto, es decir, a la efectividad de la terapia empírica inicial, incluso se ha hecho énfasis en el comienzo del tratamiento antimicrobiano lo más pronto posible, primeras 4 horas, la demora, incluso con el tratamiento adecuado, puede dar al traste con buenos resultados; el tratamiento tardío aumenta la $\operatorname{mortalidad}^{(7)}$.

Todos los consensos internacionales tienen recomendaciones similares, tanto en la clasificación de los pacientes como en el tipo de terapéutica inicial. 
Por ejemplo el grupo de pacientes que pueden llevar tratamiento ambulatorio (teniendo en cuanta sus posibilidades etiologías) la recomendación terapéutica sería betalactámicos; amoxicillina o cefalosporinas con o sin azitromicina o doxiciclina. La indicación de quinolonas respiratorias: levofloxacino, moxifloxacino, gemifloxacino, es válida, pero lo primero es que en el momento actual no contamos con ellas y lo segundo, por la experiencia adquirida de los países que la han utilizado indiscriminadamente, los niveles de resistencia del neumococo han subido rápidamente, por tal razón la mayoría de los consensos la recomiendan ante sospecha o confirmación de neumococo resistente a penicilina (NRP) ${ }^{11}$.

\section{Tratamiento ambulatorio de la NAC}

\begin{tabular}{|c|c|c|}
\hline Antimicrobianos & Dosis & Duración \\
\hline Amoxicillina & $0,5-1$ gr c/ 8 horas & 7 días \\
\hline $\mathrm{y} / \mathrm{o}$ & $\begin{array}{l}500 \mathrm{mg} \text { monodosis } \\
\text { diaria }\end{array}$ & 3 días \\
\hline Azitromicina & $\begin{array}{l}500 \mathrm{mg} \text { de entrada } \\
250 \mathrm{mg} \text { diario }\end{array}$ & 5 días \\
\hline
\end{tabular}

Sería válido aclarar la situación actual de NRP, el cual no se comporta igual en todos los continentes o países, incluso su respuesta a la penicilina varía según el lugar de la sepsis.

\section{Recomendación inicial del Clinical Laboratory Standards Institute (CLSI) para el NPR}

\begin{tabular}{lc}
\hline Sensibilidad & CIM \\
\hline Sensible & $<0,06 \mu \mathrm{g} / \mathrm{ml}$ \\
Susceptibilidad intermedia & $\begin{array}{c}0,12-1 \mu \mathrm{g} / \mathrm{ml} \\
=2 \mu \mathrm{g} / \mathrm{mL}\end{array}$ \\
Resistente & \\
\hline
\end{tabular}

En enero del 2008 se publicaron los nuevos puntos de corte para la penicilina ${ }^{(12)}$, en esta revisión desaparece el criterio de susceptibilidad intermedia para las cepas aisladas en el sistema nervioso central (SNC) y se incrementan el valor de los puntos de corte para las infecciones fuera del SNC.
Sensibilidad del neumococo fuera el SNC

\begin{tabular}{lc}
\hline Sensibilidad & CIM \\
\hline Sensible & $=2 \mu \mathrm{g} / \mathrm{mL}$ \\
Medianamente resistente & $4 \mu \mathrm{g} / \mathrm{mL}$ \\
Resistente & $=8 \mu \mathrm{g} / \mathrm{mL}$ \\
\hline
\end{tabular}

Con estos nuevos puntos de cortes, el estudio SENTRY Antimicrobial Surveillance Program en Latinoamérica, reportó en el 2008-2009 que menos del 1\% de las cepas de neumococo en infecciones respiratorias eran resistentes a penicilina. Por tal razón podemos decir que en nuestro país las infecciones respiratorias por neumococo pueden ser tratadas con altas dosis de penicilinas. Esto último nos dificultaría la aplicación de la terapia ambulatoria con penicilina natural, ya que se necesitarían dosis muy elevadas (2 millones cada 4 horas) ${ }^{(13)}$, no así con amoxicilina, que posee una alta biodisponibilidad (90\%) lo que nos facilitaría alcanzar las concentraciones necesarias, incluso por vía oral.

Con respecto a los pacientes alérgicos a la penicilina, la recomendación, basada en su espectro, sería los macrolidos (azitromicina) la cual incluye, además del neumococo, al $H$. influenzae, quien por su frecuencia no debe quedar fuera del tratamiento ${ }^{(14)}$.

Los pacientes del Grupo II, por su amplio espectro de posibilidades etiológicas y su inmunidad comprometida, deben ser tratados con distinta y más amplia antibioticoterapia. La recomendación internacional es la combinación de un betalactamico (amoxicillina con ácido clavulánico o sulbactam, cefalosporinas de segunda generación (cefuroxima) o tercera generación (ceftriaxona, cefotaxima) con un macrolido (azitromicina), de esta forma quedarían incluidos los grampositivos, gramnegativos e intraceluares (estos últimos que podrían ser los responsable hasta de un $20 \%$ de la NAC.

Observemos las siguientes recomendaciones y así podremos entender por qué nosotros no debemos utilizar los consensos que clasifican los pacientes en cinco grupos, ya que los pacientes mayores de 60 años con enfermedades asociadas que por condiciones clínicas pudieran ser tratados ambula- 
toriamente, sus posibilidades etiológicas no variarían, por tal razón tampoco lo haría la recomendación de la antibioticoterapia, y no disponemos en la farmacia de estos antibióticos mencionados (cefalosporinas 2da y 3er generación). Igual pasaría con el grupo de pacientes que podrían llevar un ingreso corto (4 días) y después con un cambio de vía terapéutica (parenteral-oral) ser egresados y completar el tratamiento ambulatorio.

Tratamiento de la NAC en sala de cuidados generales

\begin{tabular}{|c|c|c|}
\hline Antimicrobiar & Dosis & Duración \\
\hline \multicolumn{3}{|l|}{ Elección } \\
\hline Cefuroxima & $750-1500 \mathrm{mg} \mathrm{c} / 6-8$ horas & 5-10 días \\
\hline Ceftriaxona & $0,5-1$ gr c/ 12 horas & 5-10 días \\
\hline Cefotaxima & $1-2$ gr c/ $8-12$ horas & 5-10 días \\
\hline \multicolumn{3}{|l|}{$\begin{array}{l}\text { Alternativo } \\
\text { Amoxicillina/ } \\
\text { sulbactam }\end{array}$} \\
\hline \multicolumn{3}{|c|}{$\begin{array}{l}\text { Cualquiera de los anteriores } \\
\text { Con }\end{array}$} \\
\hline Azitromicina & $\begin{array}{l}500 \mathrm{mg} \text { monodosis diaria } \\
\text { ó } \\
500 \mathrm{mg} \text { de entrada } \\
250 \mathrm{mg} \text { diario }\end{array}$ & 3 días \\
\hline
\end{tabular}

En función de proteger las cefalosporinas de tercera generación frente a la creciente resistencia bacteriana, que viene siendo reportada frente a las mismas, específicamente por la aparición cada vez más frecuente de cepas productoras de betalactamas de espectro extendido(BLEE), proponemos hacer un uso cada vez más racional de la mismas y para ello proponemos utilizar como recomiendan algunos consensos ${ }^{(9-13)}$, como primera opción en el tratamiento de la NAC la cefuroxima, cefalosporina de segunda con una importante difusión al aparato respiratorio, espectro muy similar al de la cefalosporinas de tercera generación y con una alta efectividad comprobada en la NAC. El uso racional de la cefalosporinas de tercera generación redundaría en una menor aparición de BLEE ya que uno de los factores promotores de su aparición es precisamente el uso de dichas cefalosporinas ${ }^{(15)}$.

Por otra parte considero que no estaría demás, debido a la alta incidencia de su uso inadecuado, mencionar algunos antimicrobianos que no son recomendados en ninguno de los consensos internacionales ${ }^{(9,14,16-18)}$, por ejemplo; los aminoglucósidos, antibióticos con espectro reducido a gramnegativos, con pésima difusión pulmonar e inactivados en $\mathrm{Ph}$ ácido (pulmón infectado $\mathrm{Ph}=6,6$ ) y por ácidos nucleícos liberados de la muerte de leucocitos. Solo son recomendados en la neumonía grave o severa en UCI e incluso con el advenimiento de la quinolonas respiratorias han ido siendo desplazados por las mismas, por mejor espectro, más difusión y menor toxicidad.

Otro antimicrobiano, mal utilizado con frecuencia en la NAC es la ciprofloxacino, excelente miembro de la familia de las quinolonas, con potente acción antipseudomónica y frente a otras bacterias gramnegativas, pero con escaso o nula frente al neumococo, de ahí que no este contemplado dentro de las llamadas quinolonas respiratorias y su indicación en la NAC sea como los aminoglucosidos, para sinergizar betalactamicos frente a la sospecha o presencia de Pseudomona aeruginosas u otros gramnegativos.

Otro antimicrobiano, que ha tomado cierta vigencia en la actualidad es el sulfametoxazol/trimetoprim (sulfaprin), su vigencia se ha debido a la aparición y desarrollo, cada vez mas preocupante, de las cepas de $S$. aureus resistentes al meticillin de origen comunitario (SAMR.com). Este antimicrobiano a pesar de que siempre incluyó en su espectro el neumococo, en la actualidad los altos niveles de resistencia de este germen lo han excluido de la terapéutica de la NAC ${ }^{(19)}$.

Sin embargo retomando el tema de las cepas SAMR.com, las cuales son hoy la principal causa de infección de piel y partes blandas en EE.UU., estas tienen diferentes características tanto moleculares, genéticas como epidemiológicas y clínicas de las anteriormente existente cepas SAMR hospitalarias. Una de sus importantes diferencias radica en la producción de una enzima denominada Paton Valentin leucocidin, con gran efecto dermonecrótico, destructora de leucocitos y productora de necrosis pulmonar (neumonía necrotizante), cuyo grupo de riesgo principal son los niños y adolecentes, llegando a provocar complicaciones respi- 
ratorias graves en corto período de tiempo, con una mortalidad que puede llegar al $30 \%$, frecuentemente a partir de lesiones infectadas de piel. Esta cepas a diferencia de las hospitalarias, se comportan sensibles, además de a la vancomicina, frente a la clindamicina, doxiciclina, minociclina y el antimicrobiano en cuestión, sulfaprin, por tal motivo una de sus indicaciones sería en la NAC en el curso de lesiones infectadas de piel, con sospecha o aislamiento de cepas SAMR $^{(20)}$.

Respecto a la respuesta y duración del tratamiento, puede ser particularizada en cada paciente, para esto es muy importante el seguimiento de los marcadores clínicohumorales de mejoría. La observación de la respuesta inicial se hará durante las primeras 72 horas, lapso de tiempo que no esta recomendado realizar cambios en la terapéutica, debido a que como es conocido los antimicrobianos necesitan un tiempo de acción. En este período los signos clínicos que se preconiza son; caída de la fiebre, mejoría de la frecuencia respiratoria, de la frecuencia cardiaca, del apetito y mejoría del sensorio $^{(21)}$. Por la parte humoral, caída de los valores de los reactantes de fase aguda (biomarcadores): leucocitosis, eritrosedimentación, proteína $C$ reactiva y procalcitonina (no disponible) $)^{(22,23)}$.

La evolución radiológica será más lenta, razón por la cual en los pacientes que han tenido buena evolución clínica no necesariamente deben repetirse la radiografía de tórax antes del egreso(7).

La duración del tratamiento no es algo rígido, dependerá del tipo de paciente y su evolución, pero de forma general oscilará entre 5 y 10 días. La American Thoracic Society recomienda no menos de 5 días, la Britihs thoracic society siete días y el ConsenSur entre 7-10 días.

\section{Conclusiones}

Todos los consensos internacionales tienen recomendaciones similares, tanto en la clasificación de los pacientes como en el tipo de terapéutica inicial.

\section{Referencias}

1. Brown S, Jones B E, Jephson A, Dean N. (2009). Validation of the Infectious Disease Society of America/American Thoracic Society 2007 guidelines for severe community-acquired pneumonia. Critical Care Medicine, 37(12):3010-3016.

2. Valencia M, Badia J, Cavalcanti M, Ferrer M, Agustí C, Angrill J, García E, Mensa J, Niederman M, Torres A. (2007). Pneumonia Severity Index Class V Patients With Community-Acquired Pneumonia. Characteristics, Outcomes, and Value of Severity Scores CHEST, 132(2):515-522.

3. Buising KL, Thursky KA, Black JA, Mac Gregor L, Street AC, Kennedy MP, Brown' GV. (2006). A prospective comparison of severity scores for identifying patients with severe community acquired pneumonia: reconsidering what is meant by severe pneumonia. Thorax, 61:419-424.

4. Cereceda J, Maturana R. Acavedo V, Aylwin M, Flores M. (2003). Índice de gravedad en neumonía comunitaria hospitalizada. Rev Chil Enf Respir, 19:155-159.

5. Chalmers J, Singanayagam A, Hill A. (2008). Predicting the Need for Mechanical Ventilation and/or Inotropic Support for Young Adults Admitted to the Hospital with Community-Acquired Pneumonia. Clinical Infectious Diseases, 47(12):1571-1574.

6. España PP, Capelastegui A, Gorordo I, Esteban C, OribeM, Ortega M, et al. (2006). Development and validation of a clinical prediction rule for severe community-acquired pneumonia. Am J Respir Crit Care Med, 174:1249-56.

7. Brown S, Dean N. (2010). Defining and Predicting Severe Community-Acquired Pneumonia (SCAP). Curr Opin Infect Dis, 23(2):158-164.

8. Boletín de Información terapéutica para la APS en Cuba. Guía de práctica clínica para el tratamiento de la neumonía adquirida en la comunidad. Boletín No.25 Junio (2009). ISSN: 1608-7518.

9. Moreno R, Riquelme R. (2005). Etiología de la neumonía adquirida en la comunidad en el adulto inmunocompetente. Rev. Chil Enf Respir, 21:81-8.

10. Fernández R, Mauricio, Zagolin B, Mónica, Ruiz C, Mauricio et al. (2003). Neumonía adquirida en la comunidad que se hospitaliza: estudio etiológico. Rev. Méd. Chile, mayo 131(5):498-504.

11. Alós JI. Quinolonas. (2003). Rev Enfer Infecc Microb Clin, 21(5):261-268. 
12. Wayne, PA. (2008). Clinical and Laboratory Standards Institute. Performance standards for antimicrobial susceptibility testing. 18th informational supplement. CLSI document M100-S18.

13. Luna CM, Efrom ED, Schiavi y cols. (2003). Neumonía adquirida en la comunidad en adultos. Guía práctica clínica para la Argentina. Medicina, 63:319-343.

14. Díaz A, Labarca J, Pérez C, Ruiz M, Wolff M. (2005).Tratamiento de la neumonía del adulto adquirida en la comunidad. Rev Chil Enf Respir, 21:117-131.

15. Martínez P, Mattar S. (2007). Emergencia de la resistencia debido a betalactamasas de espectro extendido (BLEE) detección, impacto clínico y epidemiologia. Infectio, 11(1):23-25.

16. Lim WS. (2009). Pneumonia Guidelines Committee of the BTS Standards of Care Committee. The British Thoracic Society Guidelines for the management of Community Acquired Pneumonia in adults update. Disponible online en: www.brit-thoracic.org. uk/clinical-information/pneumonia/pneumonia-guidelines.

17. Bantar C, Bavestrello L, Curcio D, Jasovich A. y Grupo ConsenSur. (2002). Neumonía adquirida en la comunidad en adultos. Guía para la terapia empírica inicial basada en evidencia local de un grupo de trabajo sudamericano (ConsenSur). Journal of Chemotherapy, 14(4):1-30.
18. Menéndez K, Aspa J, Capelastegui A, Prat C, Rodríguez F. (2010). Normativa de neumonía adquirida en la comunidad. Madrid: Editorial Respira, pp. 23-25.

19. Agudelo CI, Castañeda E, Corso A, Regueira M, Cunto MC, Pires A, Maldonado A et al. (2009). Resistencia a antibióticos no betalactámicos de aislamientos invasores de Streptococcus pneumoniae en niños latinoamericanos. SIREVA II, 2000-2005. Rev Panam Salud Publica/Pan Am J Public Health, 25(4).

20. Daum R. (2007). Skin and Soft-Tissue Infections Caused by Methicillin-Resistant Staphylococcus aureus. $N$ Engl J Med, 357:380-90.

21. Oosterheert J, Bonten M, Schneider M, Buskens E, Lammers J, Hustinx $\mathrm{W}$ et al. (2006). Effectiveness of early switch from intravenous to oral antibiotics in severe community acquired pneumonia. Multicentre randomized trial. BMJ, 333-1193.

22. Menéndez R, Martínez R, Reyes S, Mensa J, Filella X, Marcos A y col. Biomarkers improve mortality prediction by prognostic scales in community-acquired pneumonia. Thorax 2009;64:587-591

23. Mira JP, Max A, Burgel PR. The role of biomarkers in community-acquired pneumonia: predicting mortality and response to adjunctive therapy. Critical Care 2008; 12(Suppl 6):S5 